\title{
DNA topoisomerase II $\alpha$ and RAD21 cohesin complex component are predicted as potential therapeutic targets in bladder cancer
}

\author{
ZHILING YU ${ }^{1}$, QIUPING XU ${ }^{2}$, GUIXUE WANG ${ }^{3}$, MOLLY ROWE $^{4}$, CAMERON DRISKELL $^{4}$, \\ QIAN XIE $^{2}$, MINHONG WU ${ }^{1}$ and DONGYU JIA ${ }^{3,4}$ \\ ${ }^{1}$ Department of Urinary Surgery, The People's Hospital of Yichun City, Yichun, Jiangxi 336000, P.R. China; \\ ${ }^{2}$ Morphism Institute, Seattle, WA 98117, USA; ${ }^{3}$ Key Laboratory for Biorheological Science and Technology of \\ Ministry of Education, State and Local Joint Engineering Laboratory for Vascular Implants, \\ Bioengineering College of Chongqing University, Chongqing 400044, P.R. China; \\ ${ }^{4}$ Department of Biology, Georgia Southern University, Statesboro, GA 30460, USA
}

Received October 24, 2018; Accepted April 12, 2019

DOI: $10.3892 / \mathrm{ol} .2019 .10365$

\begin{abstract}
Cancer is essentially a genetic disease. Accumulated gene mutations accelerate genome instability, which eventually leads to uncontrollable growth of the tumor. Bladder cancer is the most common form of urinary tract cancer. This form of cancer has a poor prognosis due to its clinical heterogeneity and molecular diversity. Despite recent scientific advances, the knowledge and treatment of bladder cancer still lags behind that of other types of solid tumor. In the present study, available large data portals and other studies were used to obtain clinically relevant information, and the data were systematically processed to decipher the genes associated with bladder cancer. Genes associated with the survival time of patients with bladder cancer were successfully identified. The genes were enriched in common biological processes and pathways, and upregulated in tumor samples from patients. Among the top genes identified as associated with good or poor survival in bladder cancer, DNA topoisomerase II $\alpha$ (TOP2 $\alpha$ ) and RAD21 cohesin complex component (RAD21) were also increased in bladder cancer tissues and cell lines. Therefore, TOP $2 \alpha$ and RAD21 could be used as potential therapeutic targets in bladder cancer.
\end{abstract}

\section{Introduction}

Cancer is a remarkable neoplastic disease derived from normal cells. Some normal cells are able to evolve progressively over years to reach a neoplastic state. During this period,

Correspondence to: Dr Dongyu Jia, Department of Biology, Georgia Southern University, 68 Georgia Avenue, Statesboro, GA 30460, USA

E-mail: djia@georgiasouthern.edu

Key words: DNA topoisomerase II $\alpha$, RAD21 cohesin complex component, therapeutic target, bladder cancer the cells acquire 10 hallmarks of cancer. These hallmarks include sustaining proliferative signaling, avoiding immune destruction, evading growth suppressors, tumor-promoting inflammation, resisting cell death, genome instability and mutation, enabling replicative immortality, inducing angiogenesis, deregulating cellular energetics, and activating invasion and metastasis (1). Despite these 10 characteristics, cancer is essentially a genetic disease (2,3). Accumulated mutations in specific genes results in accelerated genome instability, which eventually leads to uncontrollable growth of the tumor (1). Due to the significance of the cancer genome, The Cancer Genome Atlas (TCGA) program spent over a decade collecting the clinical data and molecular profiles of 33 distinct types of cancer based on $>11,000$ human tumors (4).

Bladder cancer is the most common form of urinary tract cancer, leading to $>165,000$ succumbing to the tumor every year worldwide $(5,6)$. Bladder cancer is a heterogeneous disease with a poor prognosis, indicating the tremendous challenge that the treatment of this disease proposes. Up until 2016, no new drugs had been approved for the treatment of bladder cancer for $>2$ decades (6). In 2014, TCGA reported comprehensive molecular profiles of bladder cancer, confirming the clinical heterogeneity and molecular diversity of the tumor (7). In 2017, TCGA presented an expanded study of bladder cancer, which included more tumor samples and linked molecular subtypes with patient outcomes (8).

Despite recent scientific advances, the knowledge and treatment of bladder cancer still lags behind that of other solid tumors (6). In the present study, publicly available large data portals and other studies were used to obtain clinically relevant information, and the data were systematically processed to decipher the genes associated with bladder cancer. The genes were enriched in common biological processes and pathways, and upregulated in tumor samples from patients. Among the top genes identified as associated with good or poor survival in bladder cancer, DNA topoisomerase II $\alpha$ (TOP2 $\alpha$ ) and RAD21 cohesin complex component (RAD21) were also enriched in bladder cancer tissues and cell lines. Therefore, TOP $2 \alpha$ and RAD21 could be used as potential therapeutic targets in bladder cancer. 


\section{Materials and methods}

PREdiction of clinical outcomes from genomic profiles (PRECOG). A research team at Stanford University (Stanford, CA, USA) developed the publicly available PRECOG portal (precog.stanford.edu) (9), which provided numerous datasets of cancer expression regarding gene expression levels and clinical outcomes. For each gene, PRECOG performed a meta- $Z$ analysis to assign a $Z$-score, which was associated with patient overall survival. Positive and negative $Z$-scores indicated poor and good survival, respectively. Z-scores of bladder cancer-associated genes were first extracted from the PRECOG database (last updated date of analysis, June 22, 2018), and ranked and analyzed using Microsoft Office 365 ProPlus Excel (version 1902; Microsoft Corporation, Redmond, WA, USA). Absolute Z-score values $>3.00$ were considered highly significant.

Gene ontology (GO) enrichment analysis. GO analysis of biological processes were performed using the publicly available GO consortium portal (geneontology.org; last updated date of analysis, June 22, 2018). The genes closely associated with overall survival time in patients with bladder cancer were studied.

SABiosciences pathway analysis. In a previous study, the publicly available SABiosciences array gene tables (qiagen.com/us/shop/genes-and-pathways/complete-biology-list/) were extracted, which provided 84 distinct literature-based curated genes that represent each molecular pathway (10). An analysis of the overlap between the bladder cancer-associated genes and the genes that represent the 67 different molecular pathways was performed.

cBioPortal. cBioPortal (cbioportal.org) was launched by the Memorial Sloan Kettering Cancer Center (New York, NY, USA) to help cancer researchers to analyze cancer genomic data $(11,12)$. In regard to bladder cancer, it provided TCGA bladder cancer dataset (8). Bladder cancer-correlated genes were inputted into the bladder cancer dataset to search for alterations in mRNA expression. Genes that were co-expressed with either TOP $2 \alpha$ or RAD21 were downloaded via the 'Co-Expression' tab, and ranked based on the Spearman Score provided by the database. cBioPortal provided free usage of its images and services (cbioportal. org/faq.jsp).

Gene expression across normal and tumor tissue (GENT). The Korea Research Institute of Bioscience and Biotechnology (Daejeon, South Korea) publicly provided and maintained the GENT portal (medical-genome.kribb.re.kr/GENT) (13). GENT included gene expression data from 16,400 samples (Affymetrix ${ }^{\circledR}$ Human Genome U133A array; 241 data sets, Thermo Fisher Scientific, Inc.) across diverse tissues. Gene expression data of the bladder cancer-correlated genes in normal and bladder cancer tissues were extracted from the GENT database (last updated date of analysis: 06/22/18), then processed in Microsoft Office 365 ProPlus Excel (version 1902; Microsoft Corporation) and GraphPad Prism (version 7; GraphPad Software, Inc., La Jolla, CA, USA).
Broad Institute Cancer Cell Line Encyclopedia (CCLE). The gene expression data of 947 human cancer cell lines were publicly provided by the CCLE (portals.broadinstitute. org/ccle), including 26 bladder cancer cell lines (14).

Statistical analysis. The data bars for the figures in the present study were produced using Microsoft Office 365 ProPlus Excel (version 1902; Microsoft Corporation), and the box and whisker plots were produced using GraphPad Prism (version 7; GraphPad Software). P-values of the datasets between normal and bladder cancer tissues were calculated using a permutation test (15). For the Kaplan-Meier plots, three Gene Expression Omnibus (GEO) series (GSE) datasets in GEO microarray database were utilized, which included GSE5479 (ncbi.nlm. nih.gov/geo/query/acc.cgi?acc=GSE5479), GSE13507 (ncbi. nlm.nih.gov/geo/query/acc.cgi?acc=GSE13507) and GSE5287 (ncbi.nlm.nih.gov/geo/query/acc.cgi?acc=GSE5287). For each dataset, the PRECOG portal (precog.stanford.edu) was used to divide patients into two groups according to the median expression of the gene. The two groups were later compared in terms of overall survival, and a survival curve was displayed. The log-rank test was utilized to test the null hypothesis of no difference between the two groups in overall survival. $P<0.05$ was considered to indicate a statistically significant difference.

\section{Results}

Genomic profiles reveal the genes strongly correlated with overall survival in bladder cancer patients. PRECOG provided 166 different datasets for different types of cancer using $\sim 18,000$ cases (9) to analyze overall survival, which indicates the length of survival time. For each gene in each type of cancer, a Meta-Z analysis with PRECOG was performed to assign a $\mathrm{Z}$-score, which was associated with the overall survival time of patients with cancer. Positive and negative Z-scores indicated poor and good overall survival, respectively. The present study focused on bladder cancer. To investigate the association between genes and the survival time of patients with bladder cancer, the Z-scores of all genes present in bladder cancer were extracted. Absolute Z-score values above 3.00 were considered highly significant. Two lists of genes strongly associated with either poor overall survival (Z-score, $\geq 3.00$ ) or good overall survival (Z-score, $\leq-3.00$ ) were obtained (data not shown). Among those genes, TOP2A, BIRC5, CDA, AURKB, CDC20, IL8, CCNA2, TMEM45A, LIPG, KPNA2, IGFL2, CXCL1, MMP11, TROAP, CENPF, MCM7, CD109, CNN3, S100A8 and LCE2D were the top 20 genes associated with poor overall survival, whereas CRTAC1, ASB12, UGT1A6, FBP1, SPINK1, SORL1, FABP4, ST3GAL5, TBC1D1, DISP1, PPP2R2B, UNC5B, MST1R, WDR88, TESC, SLC23A2, FIG4, STK32A, HIBCH and ATP8B1 were the top 20 genes associated with good overall survival (Fig. 1).

$G O$ analysis reveals the biological processes involved. To further investigate the intrinsic connections among the genes strongly associated with the overall survival of patients with bladder cancer, GO enrichment analysis of biological processes was performed. It was revealed that the poor overall survival-associated genes ( $Z$-score, $\geq 3.00$ ) were mainly 


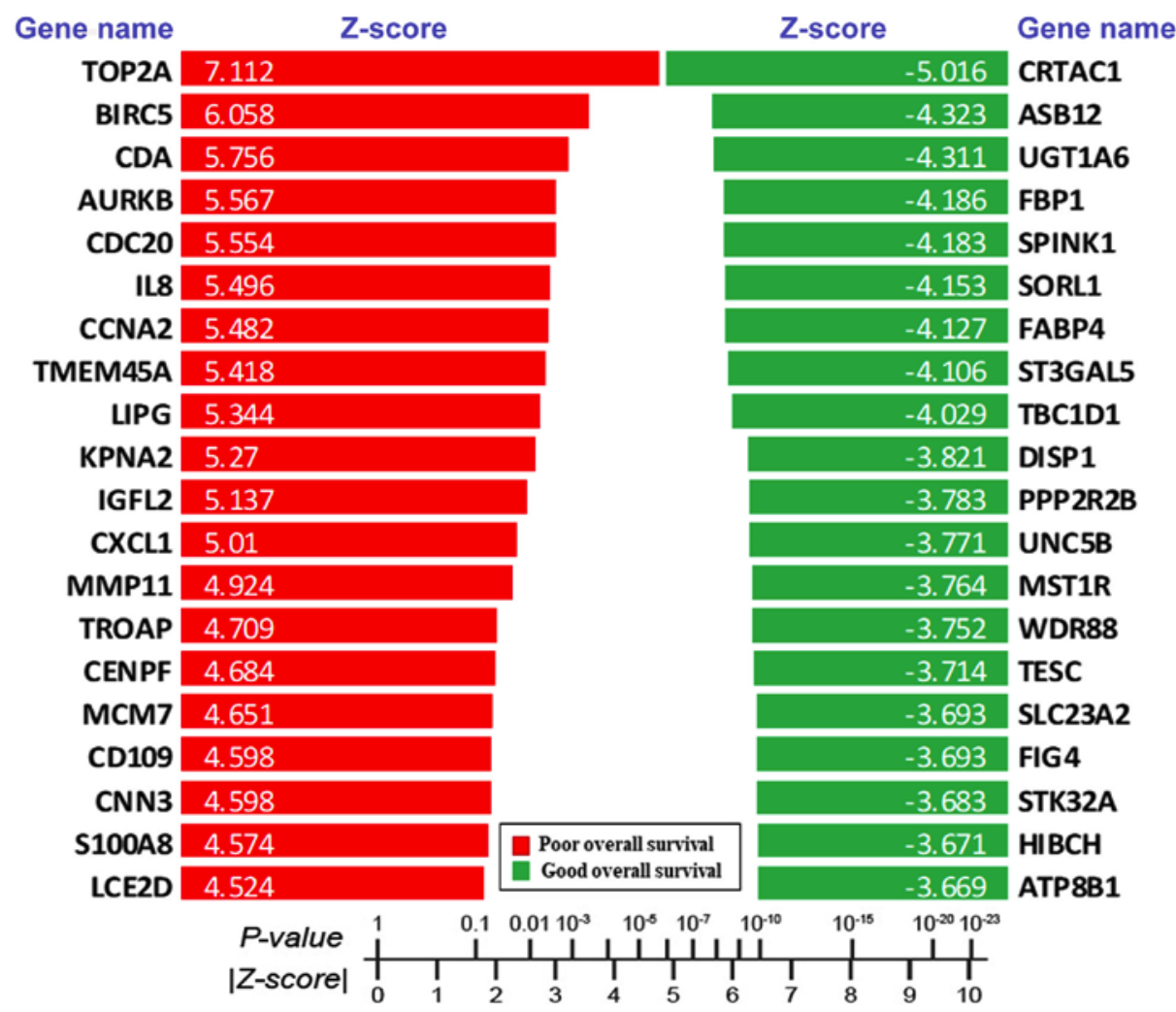

Figure 1. Z-scores of the top 20 genes correlate with poor and good overall survival in patients with bladder cancer, respectively. Using the PREdiction of Clinical Outcomes from Genomic Profiles database, the survival Z-scores of genes strongly correlated with either poor or good overall survival in bladder cancer were extracted. Note that the Z-score coincides with the P-value.

involved in cell division and the cell cycle (Fig. 2). The genes associated with good overall survival (Z-score, $\leq-3.00)$ did not show any intrinsically associated biological processes (Fig. 2). Therefore, the following sections primarily focus on the poor overall survival-correlated genes.

SABiosciences Pathway analysis reveals the pathways associated with poor overall survival in patients with bladder cancer. Since the poor overall survival-associated genes (Z-score, $\geq 3.00$ ) in bladder cancer demonstrated common biological processes, the molecular pathways that may be associated with the poor overall survival-correlated gene set were investigated. In a previous study, SABiosciences Pathway gene tables were extracted, which provided 84 distinct literature-based curated genes to represent each molecular pathway (10). In the present study, an analysis was performed assessing gene overlap between the 300 poor overall survival-associated genes (Z-score, $\geq 3.00$ ) and the genes representative of 67 different molecular pathways. The pathways identified with the largest overlap were associated with angiogenesis, cancer drug targets, cellular senescence, and mesenchymal transition (EMT), extracellular matrix and adhesion molecules, and MYC proto-oncogene, basic helix-loop-helix transcription factor (MYC) Targets (Fig. 3).

cBioPortal suggests that the mRNA levels of poor survival-correlated genes are upregulated. cBioPortal provided the bladder cancer dataset (8), which included the data of mRNA expression alterations in patients with bladder cancer. It was revealed that the mRNA levels of the
300 poor overall survival-associated genes ( $\mathrm{Z}$-score, $\geq 3.00$ ) were upregulated in 393/404 (97.3\%) of the sequenced cases/patients. This indicated the essential roles that these genes serve in promoting bladder cancer. The percentage of tumors from the patients that had upregulated mRNA for each gene were ranked from largest to smallest, and RAD21 was ranked number 1 , which was upregulated in $24 \%$ of patients with bladder cancer (Table I).

TOP $2 \alpha$ and RAD 21 are important in bladder cancer. PRECOG revealed that the Z-score of TOP2 $\alpha$ (Z-score, 7.112) was ranked number 1 , indicating the strongest association with poor overall survival in patients with bladder cancer (Fig. 1). cBioPortal ranked RAD21 number 1, as the gene was upregulated in $24 \%$ of patients with bladder cancer and was the most frequently upregulated gene in these patients (Table I). In addition, TOP $2 \alpha$ and RAD21 were among the top 50 genes of PRECOG (Z-score cut-off value, 4.07) and cBioPortal (percentage cut-off value, $9 \%$ ) analyses. A number of other genes, including BIRC5, CDA, AURKB, SUPT5H, NUF2 and ATAD2 were not selected, as they were not top genes in the two analyses, even though they were on one list of the two. Furthermore, TOP2 $\alpha$ and RAD21 were highlighted in the SABiosciences Pathway analysis (Fig. 2), which demonstrated their importance.

TOP $2 \alpha$ may be a potential therapeutic target in bladder cancer. PRECOG demonstrated that TOP $2 \alpha$ had the strongest association with poor overall survival in bladder cancer (Fig. 1), highlighting its significance. In addition, the 


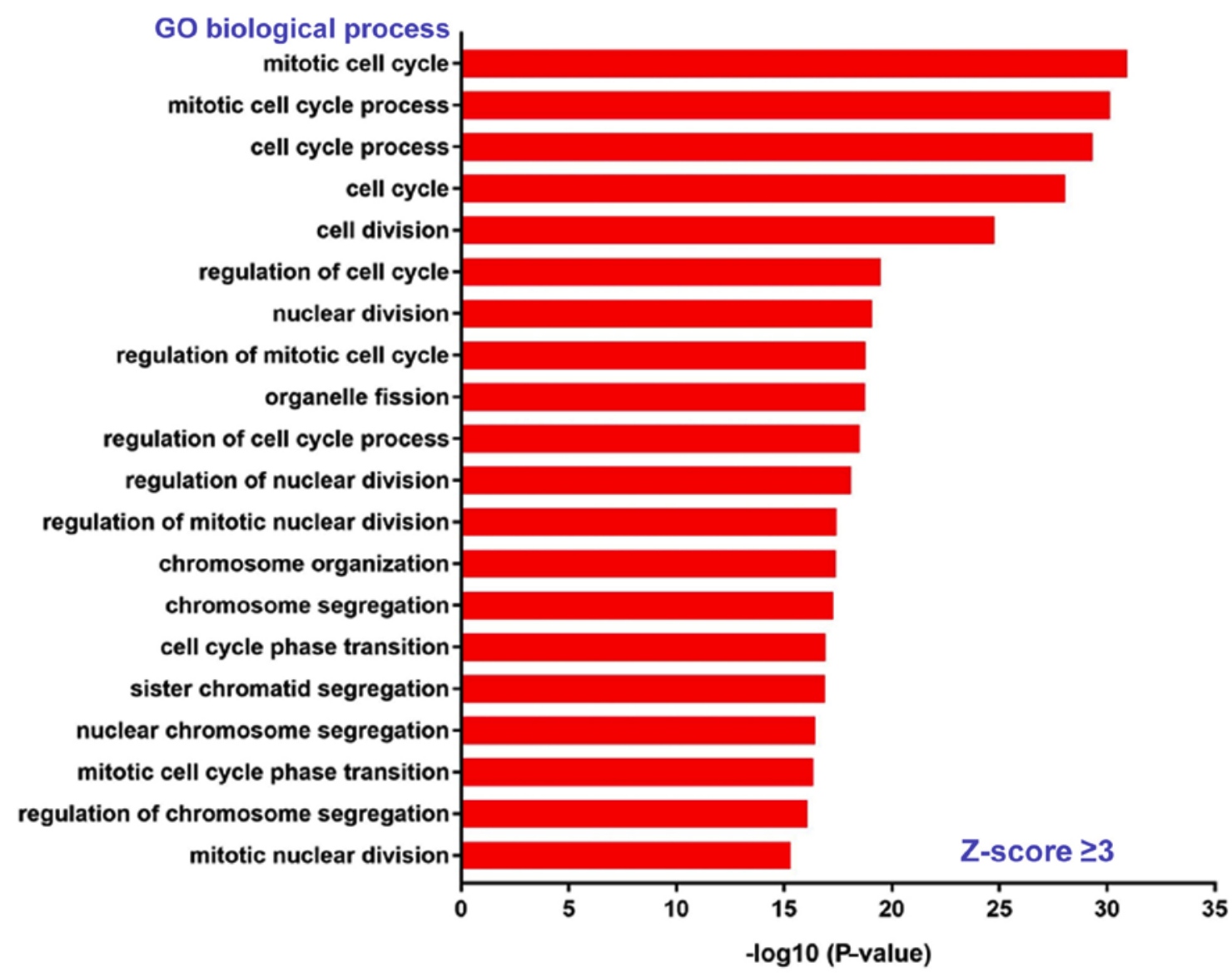

No statistically significant results found

Z-score $\leq-3$

Figure 2. Gene Ontology analysis reveals the biological processes involved with statistical significance. P-values were converted into -log10 values in terms of the reported data.

$\mathrm{GO}$ analysis of biological processes indicated that cell cycle regulation was involved (Fig. 2), and TOP2 $\alpha$ was known to be an important cell cycle regulatory gene $(16,17)$. Meanwhile, SABiosciences Pathway analysis revealed that the 300 poor overall survival-correlated genes (Z-score, $\geq 3.00$ ) had the largest overlap with the cancer drug targets-associated pathway, and TOP2 $\alpha$ was one of the overlapped genes (Fig. 3). Furthermore, cBioPortal indicated that the mRNA expression of TOP $2 \alpha$ was upregulated in $9 \%$ of patients with bladder cancer (Table I). The aforementioned results indicated the essential role of TOP $2 \alpha$ in the survival time of patients with bladder cancer and the underlying molecular mechanism.

The expression levels of TOP $2 \alpha$ in bladder cancer tissues and cell lines were further investigated. The GENT portal allowed the standardized mRNA expression of TOP $2 \alpha$ in normal bladders and corresponding bladder cancer tissues to be obtained (13). The analysis in the present study revealed that TOP $2 \alpha$ expression was significantly upregulated in bladder cancer tissues (Fig. 4A). The CCLE database included the expression data of 26 bladder cancer cell lines. It demonstrated that the mRNA level of TOP $2 \alpha$ was highly upregulated in bladder cancer cell lines (Table II). The results from the present study were consistent with a previous publication in that TOP $2 \alpha$ amplification and TOP $2 \alpha$ immunostaining were observed in bladder cancer cells and tumors (18). Meanwhile, the majority of cell lines in the present study (23/26) were derived from a transitional cell carcinoma. On the basis of the invasiveness of the carcinoma, these cell lines were classified into different stages (19). The expression levels of TOP $2 \alpha$ mRNA in Stages 1 and 2 were generally lower than those in Stages 3 and 4 , indicating that high levels of TOP $2 \alpha$ may also be associated with advanced cancer stages (Fig. 4B). In addition, bladder cancer cell line 253JBV was derived from 253J after five passages in a mouse bladder, demonstrating enhanced tumorigenicity (20). TOP $2 \alpha$ mRNA expression in 253JBV (6.538849165) was higher than in 253J (5.742801597), further implying that TOP2 $\alpha$ was associated with cancer invasiveness (Table II). Taken together, TOP $2 \alpha$ could be a potential therapeutic target in bladder cancer due to its critical role in the disease from various aspects.

$R A D 21$ as a potential therapeutic target in bladder cancer. RAD21 was also investigated due to its upregulated levels and frequency of upregulation in patients with bladder cancer (Table I). The PRECOG analysis revealed that the Z-score of RAD21 in bladder cancer was 4.29, indicating significant correlation with poor overall survival. In addition, the GO analysis of biological processes indicated that cell division, chromosome segregation and the cell cycle were all involved (Fig. 2), and RAD21 was known to be a structural component of the cohesin complex, which safeguards accurate chromosome segregation during mitotic division (21). Meanwhile, the SABiosciences Pathway analysis revealed that 


\begin{tabular}{|c|c|c|c|c|c|c|c|c|c|}
\hline SABiosciences pathway & $\begin{array}{c}\text { Number of } \\
\text { overlapping } \\
\text { genes }\end{array}$ & Genes i & individual $\mathrm{p}$ & pathways & $\begin{array}{l}\text { hat overlap } \\
\text { correla }\end{array}$ & $\begin{array}{l}\text { with the } \\
\text { ed genes }\end{array}$ & 0 bladder & ancer po & survival- \\
\hline Angiogenesis & 8 & VEGFC & TYMP & SPHK1 & FN1 & F3 & CXCL6 & $\mathrm{CXCL} 1$ & CCL2 \\
\hline Cancer Drug Targets & 8 & TOP2A & PLK4 & PLK1 & HSP90B1 & CDK 4 & BIRC5 & AURKB & AURKA \\
\hline Cellular Senescence & 7 & FN1 & COL3A1 & COL AA1 & CHEK1 & $\mathrm{COK} 4$ & CCNA2 & CALR & \\
\hline Epithelial to Mesenchymal Transition (EMT) & 7 & vCAN & TMEM132A & FN1 & FGFBP1 & DSC2 & COL5A2 & COL3A1 & \\
\hline Extracellular Matrix \& Adhesion Molecules & 7 & vCAN & TNC & SGCE & MMP11 & FN1 & COL7A1 & COLAA1 & \\
\hline MYC Targets & 7 & BCAT1 & UBE2C & PYCR1 & PPAT & ENO1 & CHEK1 & CDK4 & \\
\hline Aging & 6 & S100A9 & S100A8 & COL3A1 & COLAA1 & BUB१18 & ANXA5 & & \\
\hline Angiogenic Growth Factors & 6 & FN1 & TYMP & SPINK5 & FGFBP1 & CXCL6 & CCL2 & & \\
\hline Cardiovascular Disease & 6 & AEBP1 & POSTN & FN1 & COL3A1 & COL AA1 $_{1}$ & $\mathrm{CCL} 2$ & & \\
\hline Endothelial Cell Biology & 6 & TYMP & SPHK1 & FN1 & F3 & CCL2 & ANXA5 & & \\
\hline Protease Activated Receptor Signaling & 6 & VEGFC & F3 & CYR61 & CXCL1 & CCL2 & $\mathrm{ADCY7}$ & & \\
\hline Wound Healing & 6 & F3 & CXCL1 & COL5A2 & COL3A1 & COL.A1 & CCL2 & & \\
\hline Atherosclerosis & 5 & COL3A1 & TNC & LOLR & FN1 & $\mathrm{CCL} 2$ & & & \\
\hline Cancer Inflammation \& Immunity Crosstalk & 5 & MICB & 1001 & CXCR4 & CXCL1 & CCL2 & & & \\
\hline Fibrosis & 5 & Lox & CXCR4 & COL3A1 & CCL3 & CCL2 & & & \\
\hline Osteogenesis & 5 & soxg & FN1 & COL3A1 & COLAA1 & ANXA5 & & & \\
\hline Stress \& Toxicity Pathway Finder & 5 & PVR & HSP90B1 & CHEK1 & CCL2 & CALR & & & \\
\hline Cystic Fibrosis & 4 & S1OOAB & CXCL1 & CCL2 2 & CALR & & & & \\
\hline Growth Factors & 4 & VEGFC & TYMP & TNNT1 & CXCL1 & & & & \\
\hline Inflammasomes & 4 & PEA15 & HSP90B1 & CxCL1 & CCL2 & & & & \\
\hline Multiple Sclerosis & 4 & PHGDH & FN1 & CCL3 & $\mathrm{CCL} 2$ & & & & \\
\hline TGFB Signaling Targets & 4 & S100A8 & RAD21 & $\mathrm{FN} 1$ & $\operatorname{coc} 6$ & & & & \\
\hline WNT Signaling Targets & 4 & soxg & FOSL1 & FN1 & BIRCS & & & & \\
\hline Cancer Drug Resistance & 3 & TOP2A & CDK4 & BRCA1 & & & & & \\
\hline Cell Surface Markers & 3 & COLAA1 & $S 100 A 8$ & $\operatorname{CD69}$ & & & & & \\
\hline Cytoskeleton Regulators & 3 & $\mathrm{cr}$ & AURKB & AURKA & & & & & \\
\hline Hypoxa Signaling Pathway & 3 & Lox & F3 & ENO1 & & & & & \\
\hline IL6/STAT3 Signaling Pathway & 3 & CXCR4 & $\mathrm{CCL} 3$ & $\mathrm{CCL} 2$ & & & & & \\
\hline MAP Kinase Signaling Pathway & 3 & COLAA1 & $\mathrm{COK} 4$ & CCNA2 & & & & & \\
\hline Mesenchymal Stem Cells & 3 & soxg & COLA1 & ANXA5 & & & & & \\
\hline Nitric Oxide Signaling Pathway & 3 & TROAP & NUDT1 & FOXM1 & & & & & \\
\hline Oncogenes \& Tumor Suppressor Genes & 3 & COKN3 & COK4 & BRCA1 & & & & & \\
\hline Stem Cell & 3 & MME & COLAA1 & CCNA2 & & & & & \\
\hline Transplant Rejection & 3 & CXCR4 & CCL3 & CCL2 & & & & & \\
\hline Tumor Metastasis & 3 & MMP11 & FN1 & CXCR4 & & & & & \\
\hline Cell Motility & 2 & FAP & ACTN1 & & & & & & \\
\hline Cellular Stress Responses & 2 & ONAJB11 & CALR & & & & & & \\
\hline Drug Transporters & 2 & SLC7A5 & SLC16A1 & & & & & & \\
\hline EGF / PDGF Signaling Pathway & 2 & FN1 & COLA1 & & & & & & \\
\hline Hedgehog Signaling Pathway & 2 & ZIC2 & STK3 & & & & & & \\
\hline Induced Pluripotent Stem Cells & 2 & COLAA1 & CCNA2 & & & & & & \\
\hline Notch Signaling Pathway & 2 & ZIC2 & FOSL1 & & & & & & \\
\hline Notch Signaling Targets & 2 & TNC & soxe & & & & & & \\
\hline Oxidative Stress & 2 & NUDT1 & FOXM1 & & & & & & \\
\hline Phagocytosis & 2 & CLIC4 & CALR & & & & & & \\
\hline Type I Interferon Response & 2 & IF16 & CCL2 & & & & & & \\
\hline VEGF Signaling & 2 & VEGFC & SPHK1 & & & & & & \\
\hline Apoptosis & 1 & BIRC5 & & & & & & & \\
\hline Autophagy & 1 & CXCR4 & & & & & & & \\
\hline Cancer Stem Cells & 1 & CHEK1 & & & & & & & \\
\hline Cell Lineage Identification & 1 & ENO1 & & & & & & & \\
\hline Embryonic Stem Cells & 1 & CCNA2 & & & & & & & \\
\hline Hippo Signaling Pathway & 1 & STK3 & & & & & & & \\
\hline m TOR Signaling & 1 & VEGFC & & & & & & & \\
\hline Necrosis & 1 & PVR & & & & & & & \\
\hline NFkB Signaling Pathway & 1 & $\mathrm{cal} 2$ & & & & & & & \\
\hline Osteoporosis & 1 & COLAA1 & & & & & & & \\
\hline Skeletal Muscle: Myogenesis \& Myopathy & 1 & TNNT1 & & & & & & & \\
\hline Terminal Differentiation Markers & 1 & CXCR4 & & & & & & & \\
\hline TGFB / BMP Signaling Pathway & 1 & COL $1 A 1$ & & & & & & & \\
\hline WNT Signaling Pathway & 1 & FOSL1 & & & & & & & \\
\hline
\end{tabular}

Figure 3. Molecular pathways associated with the poor survival-correlated gene set. The number of overlapping genes between the 300 poor survival-correlated genes and common molecular pathways was provided using the SABiosciences pathway. 
Table I. Percentage of tumors from patients that have upregulated mRNA upregulation for each gene. Percentages were ordered from largest to smallest. Datasets were analyzed using the cBioPortal.

Gene mRNA upregulation, $\%$

\section{$R A D 21$}

24.00

SUPT5H

23.00

NUF2

21.00

ATAD2

19.00

RFC4, STK3, RECQL4, CEP72, TRIP13, IQGAP3

18.00

UBE2C, ORAOV1, PEA15

17.00

TPX2, DSN1

16.00

DDHD2

15.00

$U C K 2$

14.00

TRIO, SF3B2, DNAJB 11

13.00

WDR62, CDC25B, CBX2, SPAG5

12.00

RCE1, TBC1D23, SEC61G, POLQ, HIST2H4A, MYST3, DAXX

11.00

DHCR7, TULP3, MCM10, SLC2A4RG, AURKA

10.00

TOP2A, FOXM1, CDCA3, ANP32B, PYCR1, CENPA, FADS2, BCAT1, HIST2H3C,

9.00

CABLES2, GARS, PPFIA3, AP4S1, STIP1, NRM

BIRC5, NEK2, ATL2, UAP1, MTHFD2, CDK4, CIT, BRCA1, RAD54L, SOX9, SOCS7,

8.00

AARS, PHGDH, HCN3, RAD51AP1, OLFM2, CELSR3

CDC20, TMEM45A, KPNA2, MCM7, HES2, PTMS, VSTM2L, TMEM132A, RCOR1,

7.00

SLC4A2, SLC16A1, LMO7, ANLN, MLLT11, ASPM, CDC6, CKAP2L, NMU, PSRC1, F3,

FANCB, FAM50A, WDHD1, TNNT1, MICB, DAD1, PSORS1C2, GP6, SMC1B, HIST1H2BK, MYO10

IL8, MMP11, CENPF, CNN3, HIST1H2AM, BUB1, GPR68, SGCE, PVR, PRSS8, TK1, KIFC1,

6.00

CPA4, CNTD2, HOXC6, KIF2C, S100A9, HIST1H2BJ, EZH2, CDCA8, CDT1, NUDT1, NDE1,

DTL, PDIA4, TYMP, HPRT1, PI3, PTPN12, CRLF3, USP13, RRM2, FOSL1

AURKB, CCNA2, TROAP, PPP2R2C, LDLR, CD164L2, SPTBN1, SHCBP1, KNTC1, TMEM52,

KLHDC7B, ELFN2, CTHRC1, ADM2, CHEK1, HIST1H2BG, CP, ZBED2, PPAT, NUAK1, FGFBP1, COL7A1, ASF1B, AP2S1, STX2, HIST1H3H, MME, HIST1H2BD, SLC7A5, POLE, DNM1, CDCA2, SKA1, PLK1, CDCA5, PRSS21, POSTN, MT1E, MCM5, AGTRAP, MAD2L2, GNG4, ACTB, VCAN, SLC26A6, TRPM2, CALR, TTK, ADCY7, IRF2BP2

LIPG, CXCL1, CD109, S100A8, MYO1B, SCEL, PLK4, HOXC9, HMMR, CLIC4, CDKN3, PHF19,

PKD1L2, HIST1H2BF, VEGFC, PKMYT1, HSP90B1, JPH1, IDO1, TNC, MRPL11, FAP, LRP8,

HIST1H1C, CENPE, MKI67, GLT25D1, ANXA5, B4GALNT1, CLCF1, HIST1H4H, ACTN1,

ENO1, FN1, SPHK1, AFAP1, KLK7, HSD11B2, DUSP14, RCN3, AEBP1, MAP4K2, WDR16,

MAP4K4, PAGE2, TNFAIP8L1, E2F2, PLOD1

CDA, SPINK5, TNFRSF19, MCTP1, HIST2H2AC, CYP26B1, NUSAP1, NCAPG, ABL2,

3.00

HIST1H4E, EFCAB3, TIMELESS, TUBB2A, CYR61, BUB1B, CCL2, NFIL3, PTTG1, KIF11,

IFI6, LMF2, CCL3, CD69

S100A7, C1QTNF6, CHN1, COL5A2, DCBLD2, CTSL2, ENO2, MFAP2, FLNA

MIA, SERPINE2, DPRX, SPRRIB, PAGE2B

2.70

CCRN4L, NNMT, LOX, BAAT, CXCL6, DSC2

2.50

CXCR4, KRT4, COL1A1, FBXO5, NEK6, HIST1H4B, PALLD

2.20

CEP55, PLUNC, LGALSI

2.00

COMTD1, COL3A1, ZIC2, HBQ1, GPRIN1

1.70

IGFL2, WFDC12, KLK6

1.50

CRMP1, GFPT2

1.20

GRIN3B, GAST

1.00

LCE2D

0.70

MT1H, GPR6

0.50

SHISA8

0.00

LOC 375010

N/A

N/A, not applicable. 
A

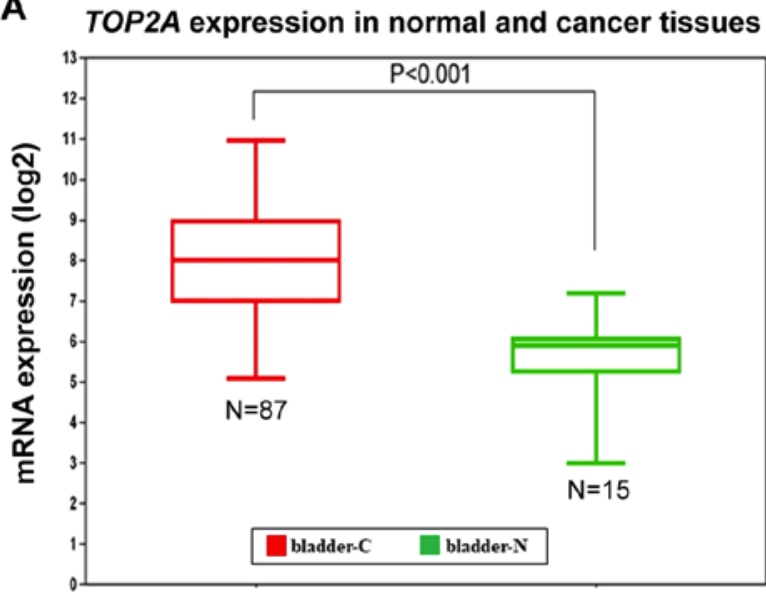

B TOP2A expression in bladder cancer cell lines of different stages

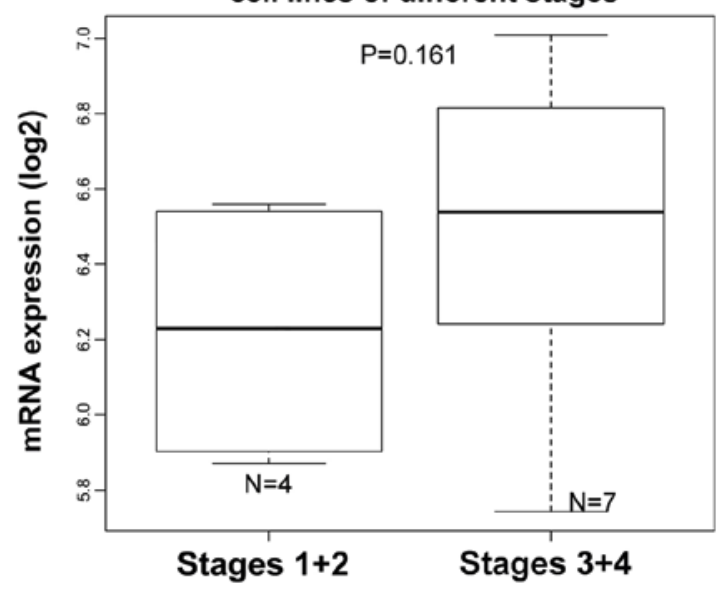

Figure 4. mRNA expression levels of TOP $2 \alpha$ in normal bladders and corresponding bladder cancer tissues, and in bladder cancer cell lines. (A) TOP2 $\alpha$ mRNA expression data from normal bladders and bladder cancer tissues. (B) The fold change of TOP $2 \alpha$ mRNA expression in bladder cancer cell lines of different stages. TOP2 $\alpha$, topoisomerase II $\alpha$; RAD21, RAD21 cohesin complex component; bladder-C, bladder cancer tissues; bladder-N, normal bladder tissues.

RAD21 was one of the four poor overall survival-correlated genes (S100A8 Z-score, 4.57; RAD21 Z-score, 4.29; FN1 $\mathrm{Z}$-score, 3.25; CDC6 Z-score, 3.47) that overlapped with the transforming growth factor- $\beta$ (TGF- $\beta$ ) signaling target-associated pathway (Fig. 3). Similarly to the function of TOP $2 \alpha$, RAD21 served an important role in the survival time of patients with bladder cancer and the underlying molecular mechanism.

The GENT analysis further demonstrated that RAD21 expression was significantly upregulated in bladder cancer tissues (Fig. 5A). The CCLE data demonstrated that the mRNA level of RAD21 was highly upregulated in bladder cancer cell lines (Table II). The expression levels of RAD21 mRNA in Stages 1 and 2 were generally lower than those in Stages 3 and 4 , indicating that high expression of RAD2 might also be associated with advanced cancer stages (Fig. 5B). In addition, RAD21 mRNA expression in the tumorigenicity-enhanced 253JBV (5.731007386) was increased compared with the original 253J (4.59366421), further indicating that RAD21 could be associated with cancer invasiveness (Table II). Subsequently, RAD21 could be another potential therapeutic target in bladder cancer.

Further validation of TOP $2 \alpha$ and RAD21 in specific types of bladder cancer. Bladder cancer is a heterogeneous disease, and many databases included all types of bladder cancer for analysis. Therefore, when data were analyzed through those databases in the present study, the type of bladder cancer was not considered. Bladder cancer has three main types: Transitional cell carcinoma (TCC), adenocarcinoma and squamous cell carcinoma (22). Prognosis of TCC may be different to that of adenocarcinoma and squamous cell carcinoma. However, due to the fact that TCC is the most common ( $95 \%$ cases) bladder cancer in the Western world (23) and most samples in the databases are from patients with TCC, this analysis is considered robust, particularly for TCC. In addition, TCC can be further classified into non-muscle-invasive, muscle-invasive and metastatic bladder cancer on the basis of tissue invasiveness (24). In order to confirm the robustness of the results from the present study, the stratified data were further analyzed. To study the relationship between TOP $2 \alpha$ mRNA expression and overall survival, three Gene Expression Omnibus (GEO) series (GSE) datasets in GEO microarray database were utilized (9), which included GSE5479 (25), GSE13507 (26) and GSE5287 (23). The patients from the GSE5479 and GSE13507 studies had non-muscle invasive bladder cancer. The stages of non-muscle invasive bladder cancer are mainly early stages Ta and T1 (27). GSE5479 and GSE13507 consistently demonstrated that high levels of TOP $2 \alpha$ were significantly associated with poor overall survival in patients with early stage bladder cancer (Fig. 6A). In patients with advanced bladder cancer (GSE5287), high expression of TOP2 $\alpha$ was still significantly associated with poor overall survival (Fig. 6A), indicating that high expression of TOP $2 \alpha$ was a poor indicator of survival time in patients with early and advanced stages of bladder cancer. RAD21 was then investigated using GSE13507 (26), GSE5287 (23) and GSE1827 (27). RAD21 was generally associated with poor overall survival in patients with early and advanced stage bladder cancer, but was not statistically significant (Fig. 6B). Interestingly, in patients with cancer that had mixed stages (GSE1827), RAD21 was significantly linked with overall survival (Fig. 6B). These results suggested that further datasets may be required in the future in order to reach a statistical confirmation of the role that RAD21 serves in specific types of bladder cancer. Furthermore, TOP $2 \alpha$ may be an improved poor overall survival indicator compared with RAD21.

\section{Discussion}

The present study of genomic profiles revealed the genes strongly associated with either poor or good overall survival in patients with bladder cancer (Fig. 1). This provided a list of genes that may be beneficial for therapeutic purposes in bladder cancer. Further GO enrichment analysis of biological processes indicated that the majority of poor survival-associated genes were involved in the cell cycle and cell division (Fig. 2). Cell cycle and cell division are critical for normal cell proliferation, and dysregulation inevitably leads 
Table II. mRNA expression levels of RAD21 and TOP2 $\alpha$, types of bladder cancer, stages and grades of the 26 Cancer Cell Line Encyclopedia bladder cancer cell lines.

\begin{tabular}{lccll}
\hline Cell line & TOP2 $\alpha$ expression, $\log 2$ & RAD21 expression, $\log 2$ & Bladder cancer types & Stage \\
\hline UMUC3 & 6.958127687 & 7.104728151 & Transitional cell carcinoma & T2-T4 \\
5637 & 6.933115661 & 5.715972879 & Transitional cell carcinoma & Unknown \\
UBLC1 & 6.27221173 & 7.058982787 & Transitional cell carcinoma & $\geq$ T2 \\
J82 & 6.419099166 & 5.874719972 & Transitional cell carcinoma & T3 \\
T24 & 7.00935111 & 5.955957403 & Transitional cell carcinoma & Ta \\
253J & 5.742801597 & 4.59366421 & Transitional cell carcinoma & T4 \\
253JB & 6.538849165 & 5.731007386 & Transitional cell carcinoma & T4 \\
HT1197 & 5.935498163 & 5.244994094 & Transitional cell carcinoma & T2 \\
UMUC1 & 5.149636116 & 5.933561268 & Transitional cell carcinoma & Unknown \\
TCCSUP & 5.408029843 & 5.407907222 & Transitional cell carcinoma & Unknown \\
JMSU1 & 7.008411182 & 6.030082453 & Transitional cell carcinoma & T4 \\
SW780 & 6.672841278 & 5.952421068 & Transitional cell carcinoma & Unknown \\
HT1376 & 6.192540552 & 6.376601431 & Transitional cell carcinoma & $\geq$ T2 \\
RT4 & 6.558977198 & 5.836951145 & Transitional cell carcinoma & T1 \\
RT112 & 6.820675745 & 6.461052848 & Transitional cell carcinoma & Ta \\
SW1710 & 6.186105774 & 5.158323384 & Transitional cell carcinoma & Unknown \\
VMCUB1 & 6.859208595 & 6.603328874 & Transitional cell carcinoma & Unknown \\
CAL29 & 6.523430466 & 5.670346132 & Transitional cell carcinoma & T2 \\
BFTC905 & 6.062034606 & 5.951141774 & Transitional cell carcinoma & T4 \\
KU1919 & 6.903279213 & 6.368675102 & Transitional cell carcinoma & T3 \\
BC3C & 6.7273538 & 6.012404086 & Transitional cell carcinoma & T3 \\
639V & 7.007183002 & 6.383729997 & Transitional cell carcinoma & Unknown \\
647V & 5.869869325 & 5.318748418 & Transitional cell carcinoma & T2/3a \\
SCABER & 5.696395585 & 5.803349542 & Squamous cell carcinoma & Not applicable \\
KMBC2 & 6.213242638 & 5.352501752 & Bladder carcinoma & Unknown \\
HS172T & 5.951000331 & 5.638518222 & Bladder carcinoma & Unknown \\
\hline
\end{tabular}

to the progression of cancer. The results of the present study indicated that poor overall survival-correlated genes possibly contribute to 4 of the 10 cancer hallmarks: Evading growth suppressors, resisting cell death, genome instability and mutation, and enabling replicative immortality. Surprisingly, genes associated with good overall survival did not indicate any intrinsically-associated biological processes (Fig. 2), demonstrating the complex molecular mechanism underlying the development of bladder cancer. Therefore, the present study primarily focused on the common features of poor overall survival-associated genes.

The SABiosciences Pathway analysis identified the largest overlap between the 300 poor overall survival-associated genes and several molecular pathways, including pathways associated with angiogenesis, cancer drug targets, cellular senescence, EMT, extracellular matrix and adhesion molecules, and MYC Targets (Fig. 3). The pathway associated with angiogenesis evidently contributed to the cancer hallmark: Inducing angiogenesis (1). The pathway associated with cancer drug targets probably contributed to all 10 cancer hallmarks (28). The pathway associated with cellular senescence likely contributed to the cancer hallmark: Resisting cell death (29). The pathway associated with EMT may contribute to two cancer hallmarks: Sustaining proliferative signaling and activating invasion and metastasis $(30,31)$. The oncogene MYC had broad effects on the initiation and progression of cancer, and the pathway associated with MYC Targets most likely contributed to all cancer hallmarks (32).

Further cBioPortal analysis demonstrated that the mRNA levels of the poor overall survival-associated genes were frequently upregulated in bladder tumors, and RAD21 was the most frequent upregulation. TOP $2 \alpha$ and RAD21 are two potential therapeutic targets in bladder cancer; the two genes were frequently upregulated in patients with bladder cancer, were significantly correlated with poor overall survival, were enriched in common biological processes and molecular pathways (Figs. 2 and 3), and were highly upregulated in bladder cancer tissues and cell lines (Figs. 4 and 5). The limitation of the present study is that the CCLE database did not provide the expression data of normal bladder cells for comparison with those of the 26 bladder cancer cell lines. Normal bladder cell lines are required for future research and necessary for databases to elucidate the differential expressions between cancer and normal cell lines.

Previous studies have demonstrated that TOP $2 \alpha$ was frequently amplified in bladder tumors (1.5\%), particularly in advanced stage tumors $(3.4 \%)(18,33)$. A recent study also indicated that TOP $2 \alpha$ amplification was associated 
A RAD21 expression in normal and cancer tissues

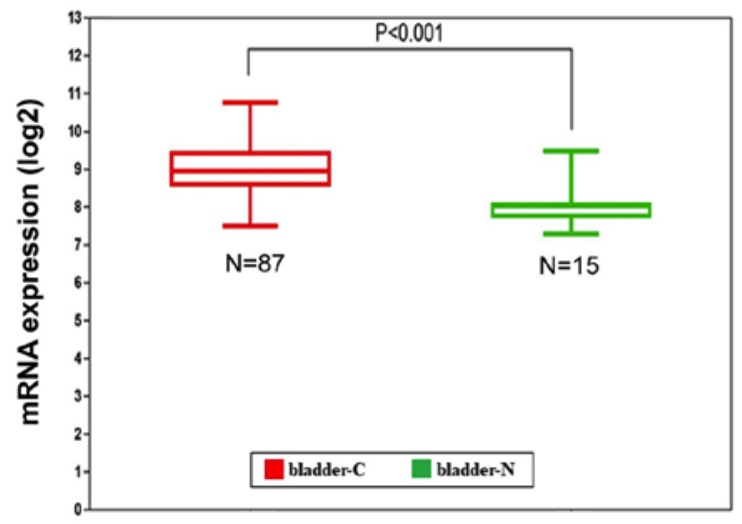

B RAD21 expression in bladder cancer cell lines of different stages

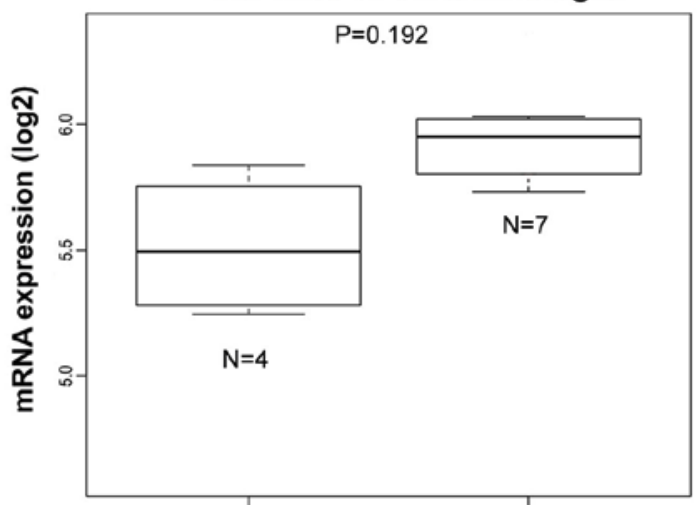

Stages $1+2$

Stages $3+4$

Figure 5. mRNA expression levels of RAD21 in normal bladders and corresponding bladder cancer tissues, and in bladder cancer cell lines. (A) RAD21 mRNA expression data from normal bladders and bladder cancer tissues. (B) The fold change of RAD21 mRNA expression in bladder cancer cell lines of different stages. TOP2 $\alpha$, topoisomerase II $\alpha$; RAD21, RAD21 cohesin complex component; bladder-C, bladder cancer tissue; bladder-N, normal bladder tissues.
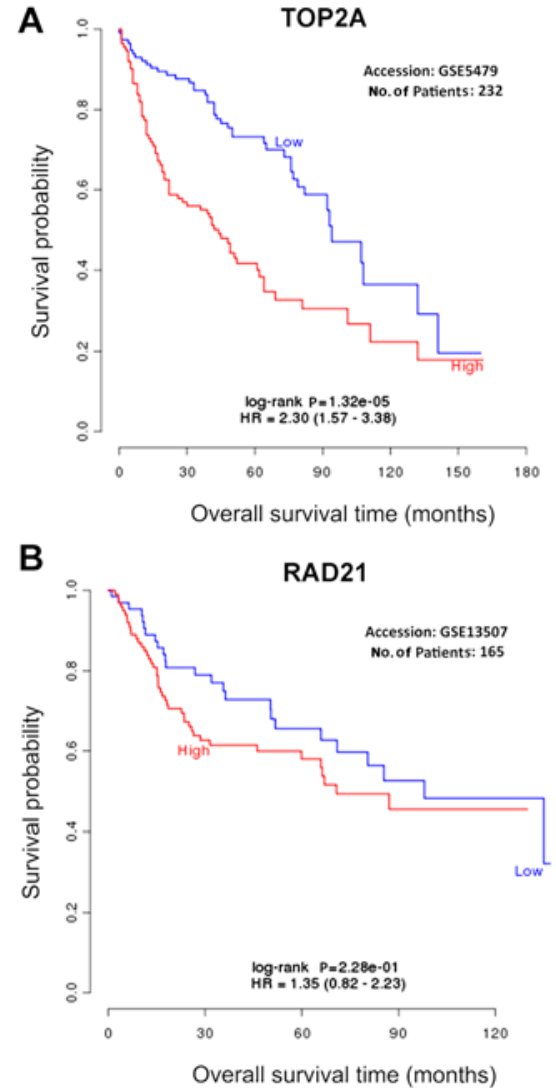
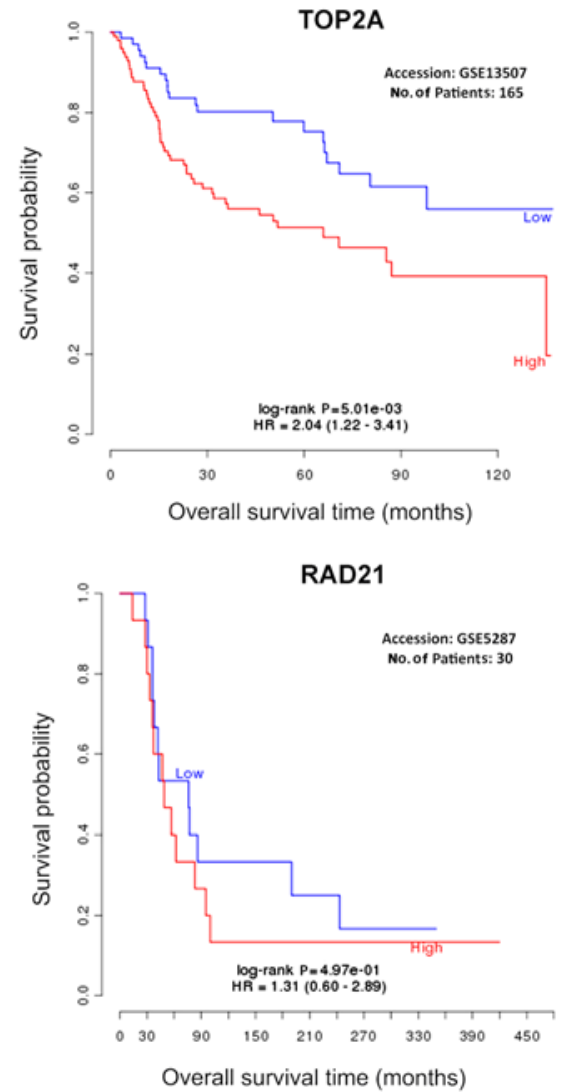

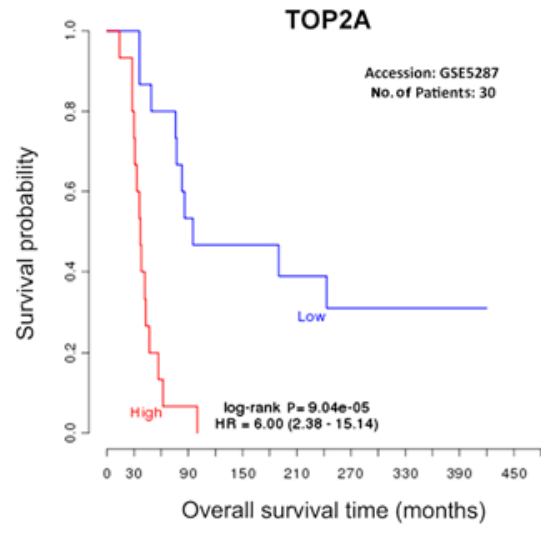

RAD21

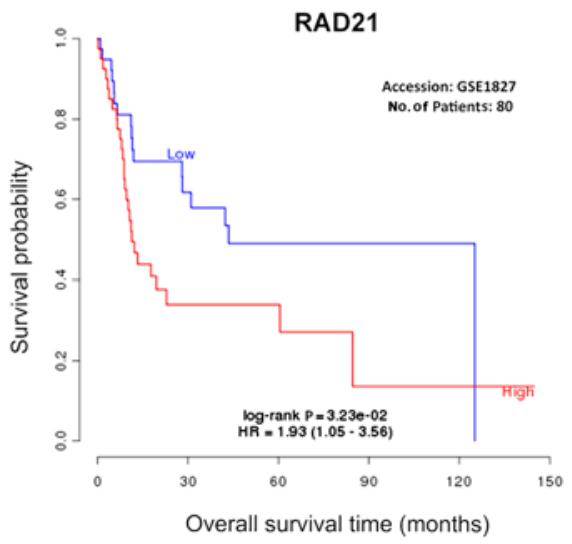

Figure 6. Association between TOP2 $\alpha$ and RAD21 with overall survival in specific types of bladder cancer. (A) The association of TOP2 $\alpha$ with overall survival. (B) The association of RAD21 with overall survival. TOP2 $\alpha$, topoisomerase II $\alpha$; RAD21, RAD21 cohesin complex component; HR, hazard ratio.

with the biological aggressiveness of upper tract urothelial carcinoma (34). Meanwhile, TOP2 $\alpha$ amplification was also observed in a number of other types of cancer, including colorectal cancer (35), breast cancer (36,37), leukemia (38) and prostate cancer (39). These studies highlighted the significance of TOP $2 \alpha$ not only in bladder cancer, but also in other types of cancer. Notably, the chromosome location of the TOP $2 \alpha$ gene is near the human epidermal growth factor receptor-2 (HER-2) oncogene, and TOP $2 \alpha$ is frequently co-amplified with
HER-2 (33). The molecular mechanism underlying how TOP $2 \alpha$ promotes tumor progression involved the induction of oncogene rearrangement (40). The more invasive a tumor becomes as a result of the rearrangements eventually leads to poor patient survival. TOP $2 \alpha$ encodes the vital enzyme topoisomerase II $\alpha$, which manages DNA topology. This enzyme can be targeted by numerous clinically available anticancer drugs, that are known to be topoisomerase II (or TopoII) inhibitors (41). 
There are currently very few studies that focus on the relationship between RAD21 and bladder cancer. Overexpression of RAD21 was only implicated in bladder cancer in a large-scale meta-analysis (42). However, RAD21 was identified as being important for many other cancers, including cervical cancer (43), ovarian cancer (44) and oral squamous cell carcinoma (45). The molecular mechanism by which high levels of RAD21 promote tumor progression may involve increased genomic instability (46) and insensitivity to chemotherapeutic drugs (47). Direct knockdown of RAD21 in cancer cell lines has been revealed to enhance anticancer effects (47).

Bladder cancer is a heterogeneous disease, and many databases include all types of bladder cancer for analysis. Therefore, the type of bladder cancer was not initially considered in the present study when analyzing the data through those databases. However, our analysis remains robust, particularly for TCC, as TCC is the most common (95\% of all cases) bladder cancer in the Western world, and the majority of samples in the databases are taken from patients with TCC. In order to confirm the robustness of the results from the present study, the stratified data were further analyzed, resulting in their validation. The present study also indicated that TOP $2 \alpha$ may be a better indicator of poor overall survival than RAD21. In the present study, the search for co-expressed genes revealed that there were 169 and 20 genes co-expressed with TOP $2 \alpha$ and RAD21, respectively (Spearman score, $\geq 0.6$ ). GO enrichment analysis of the biological processes of the TOP $2 \alpha$-co-expressed genes revealed many cell cycle-associated processes. The most significant processes included the cell cycle, the mitotic cell cycle, the cell cycle process, the mitotic cell cycle process and cell division. Similarly, GO enrichment analysis of the biological processes of the RAD21-co-expressed genes revealed the cell cycle process and the cell cycle to be the most significant processes. The results from the present study indicated that cell cycle regulatory genes are critical for the development of bladder cancer. The therapeutic targeting strategies aimed towards bladder cancer should take into account the restoration of proper cell cycle regulation. The authors hope that the present study will shed new light on the targeted therapy and drug development for the treatment of bladder cancer.

\section{Acknowledgements}

The authors would like to thank the research assistance provided by Georgia Southern University (Statesboro, GA, USA), The People's Hospital of Yichun City (Yichun, China), Morphism Institute (Seattle, WA, USA), and Chongqing University (Chongqing, China).

\section{Funding}

DJ is supported by the Start-Up funding and College of Science and Mathematics Research Grant from Georgia Southern University, the Visiting Scholar Foundation of Key Laboratory of Biorheological Science and Technology (Chongqing University), Ministry of Education (grant no. CQKLBST-2016-004). MR is supported by the Undergraduate Research Travel Award from Georgia Southern University. GW is supported by a grant from the National Natural Science Foundation of China (grant no. 11572064).

\section{Availability of data and materials}

The datasets generated and/or analyzed during the present study are available in the Research Data Repositories, figshare. com/s/746142f95a8afc151acf.

\section{Authors' contributions}

DJ designed and performed the analysis. ZY, MR, CD and MW participated in the data collection. QPX, GW and QX participated in the data analysis. DJ produced the figures and wrote the paper. All authors contributed to the paper revision. All authors approved the manuscript for submission.

\section{Ethics approval and consent to participate}

Not applicable.

\section{Patient consent for publication}

Not applicable.

\section{Competing interests}

The authors declare that they have no competing interests.

\section{References}

1. Hanahan D and Weinberg RA: Hallmarks of cancer: The next generation. Cell 144: 646-674, 2011.

2. Vogelstein B and Kinzler KW: Cancer genes and the pathways they control. Nat Med 10: 789-799, 2004.

3. Knudson AG: Two genetic hits (more or less) to cancer. Nat Rev Cancer 1: 157-162, 2001.

4. Liu J, Lichtenberg T, Hoadley KA, Poisson LM, Lazar AJ, Cherniack AD, Kovatich AJ, Benz CC, Levine DA, Lee AV, et al: An integrated TCGA pan-cancer clinical data resource to drive high-quality survival outcome analytics. Cell 173: 400-416.e11, 2018.

5. Cheung G, Sahai A, Billia M, Dasgupta P and Khan MS: Recent advances in the diagnosis and treatment of bladder cancer. BMC Med 11: 13, 2013.

6. Hurst CD and Knowles MA: Bladder cancer: Multi-omic profiling refines the molecular view. Nat Rev Clin Oncol 15: 203-204, 2018.

7. Cancer Genome Atlas Research Network: Comprehensive molecular characterization of urothelial bladder carcinoma. Nature 507: 315-322, 2014.

8. Robertson AG, Kim J, Al-Ahmadie H, Bellmunt J, Guo G, Cherniack AD, Hinoue T, Laird PW, Hoadley KA, Akbani R, et al: Comprehensive molecular characterization of muscle-invasive bladder cancer. Cell 171: 540-556.e25, 2017

9. Gentles AJ, Newman AM, Liu CL, Bratman SV, Feng W, Kim D, Nair VS, Xu Y, Khuong A, Hoang CD, et al: The prognostic landscape of genes and infiltrating immune cells across human cancers. Nat Med 21: 938-945, 2015.

10. Jia D, Liu Z, Deng N, Tan TZ, Huang RY, Taylor-Harding B, Cheon DJ, Lawrenson K, Wiedemeyer WR, Walts AE, et al: A COL11A1-correlated pan-cancer gene signature of activated fibroblasts for the prioritization of therapeutic targets. Cancer Lett 382: 203-214, 2016.

11. Cerami E, Gao J, Dogrusoz U, Gross BE, Sumer SO, Aksoy BA, Jacobsen A, Byrne CJ, Heuer ML, Larsson E, et al: The cBio cancer genomics portal: An open platform for exploring multidimensional cancer genomics data. Cancer Discov 2: 401-404, 2012.

12. Gao J, Aksoy BA, Dogrusoz U, Dresdner G, Gross B, Sumer SO, Sun Y, Jacobsen A, Sinha R, Larsson E, et al: Integrative analysis of complex cancer genomics and clinical profiles using the cBioPortal. Sci Signal 6: pl1, 2013. 
13. Shin G, Kang TW, Yang S, Baek SJ, Jeong YS and Kim SY: GENT: Gene expression database of normal and tumor tissues. Cancer Inform 10: 149-157, 2011.

14. Barretina J, Caponigro G, Stransky N, Venkatesan K, Margolin AA, Kim S, Wilson CJ, Lehár J, Kryukov GV, Sonkin D, et al: The cancer cell line encyclopedia enables predictive modelling of anticancer drug sensitivity. Nature 483: 603-607, 2012.

15. Taylor AB and MacKinnon DP: Four applications of permutation methods to testing a single-mediator model. Behav Res Methods 44: 806-844, 2012.

16. Mjelle R, Hegre SA, Aas PA, Slupphaug G, Drabløs F, Saetrom P and Krokan HE: Cell cycle regulation of human DNA repair and chromatin remodeling genes. DNA Repair (Amst) 30: 53-67, 2015

17. Park JE, Yi H, Kim Y, Chang $\mathrm{H}$ and Kim VN: Regulation of Poly(A) tail and translation during the somatic cell cycle. Mol Cell 62: 462-471, 2016.

18. Simon R, Atefy R, Wagner U, Forster T, Fijan A, Bruderer J, Wilber K, Mihatsch MJ, Gasser T and Sauter G: HER-2 and TOP $2 \mathrm{~A}$ coamplification in urinary bladder cancer. Int $\mathrm{J}$ Cancer 107: 764-772, 2003.

19. Zuiverloon TCM, de Jong FC, Costello JC and Theodorescu D: Systematic review: Characteristics and preclinical uses of bladder cancer cell lines. Bladder Cancer 4: 169-183, 2018.

20. Dinney CP, Fishbeck R, Singh RK, Eve B, Pathak S, Brown N, Xie B, Fan D, Bucana CD, Fidler IJ, et al: Isolation and characterization of metastatic variants from human transitional cell carcinoma passaged by orthotopic implantation in athymic nude mice. J Urol 154: 1532-1538, 1995.

21. Morales $\mathrm{C}$ and Losada A: Establishing and dissolving cohesion during the vertebrate cell cycle. Curr Opin Cell Biol 52: 51-57, 2018.

22. Kaufman DS, Shipley WU and Feldman AS: Bladder cancer. Lancet 374: 239-249, 2009.

23. Als AB, Dyrskjøt L, von der Maase H, Koed K, Mansilla F, Toldbod HE, Jensen JL, Ulhøi BP, Sengeløv L, Jensen KM and Orntoft TF: Emmprin and survivin predict response and survival following cisplatin-containing chemotherapy in patients with advanced bladder cancer. Clin Cancer Res 13: 4407-4414, 2007.

24. Apolo AB, Vogelzang NJ and Theodorescu D: New and promising strategies in the management of bladder cancer. Am Soc Clin Oncol Educ Book: Jun 1, 2015 (Epub ahead of print) doi: 10.14694/EdBook_AM.2015.35.105.

25. Dyrskjøt L, Zieger K, Real FX, Malats N, Carrato A, Hurst C, Kotwal S, Knowles M, Malmström PU, de la Torre M, et al: Gene expression signatures predict outcome in non-muscle-invasive bladder carcinoma: A multicenter validation study. Clin Cancer Res 13: 3545-3551, 2007.

26. Kim WJ, Kim EJ, Kim SK, Kim YJ, Ha YS, Jeong P, Kim MJ, Yun SJ, Lee KM, Moon SK, et al: Predictive value of progression-related gene classifier in primary non-muscle invasive bladder cancer. Mol Cancer 9: 3, 2010.

27. Blaveri E, Simko JP, Korkola JE, Brewer JL, Baehner F, Mehta K, Devries S, Koppie T, Pejavar S, Carroll P and Waldman FM: Bladder cancer outcome and subtype classification by gene expression. Clin Cancer Res 11: 4044-4055, 2005.

28. Dobbin ZC, Katre AA, Steg AD, Erickson BK, Shah MM, Alvarez RD, Conner MG, Schneider D, Chen D and Landen CN: Using heterogeneity of the patient-derived xenograft model to identify the chemoresistant population in ovarian cancer. Oncotarget 5: 8750-8764, 2014.

29. Wang E: Senescent human fibroblasts resist programmed cell death, and failure to suppress bcl 2 is involved. Cancer Res 55 : 2284-2292, 1995.

30. Gal A, Sjöblom T,Fedorova L, Imreh S, Beug H and Moustakas A: Sustained TGF beta exposure suppresses Smad and non-Smad signalling in mammary epithelial cells, leading to EMT and inhibition of growth arrest and apoptosis. Oncogene 27: 1218-1230, 2008.

31. Cao QH, Liu F, Li CZ, Liu N, Shu M, Lin Y, Ding L and Xue L: Testes-specific protease 50 (TSP50) promotes invasion and metastasis by inducing EMT in gastric cancer. BMC Cancer 18: 94, 2018.
32. Stine ZE, Walton ZE, Altman BJ, Hsieh AL and Dang CV: MYC, metabolism, and cancer. Cancer Discov 5: 1024-1039, 2015.

33. Järvinen TA and Liu ET: Topoisomerase IIalpha gene (TOP2A) amplification and deletion in cancer-more common than anticipated. Cytopathology 14: 309-313, 2003.

34. Aumayr K, Klatte T, Neudert B, Birner P, Shariat S, Schmidinger M, Susani M and Haitel A: HER2 and TOP2A gene amplification and protein expression in upper tract urothelial carcinomas. Pathol Oncol Res 24: 575-581, 2018.

35. Al-Kuraya K, Novotny H, Bavi P, Siraj AK, Uddin S, Ezzat A, Sanea NA, Al-Dayel F, Al-Mana H, Sheikh SS, et al: HER2, TOP2A, CCND1, EGFR and C-MYC oncogene amplification in colorectal cancer. J Clin Pathol 60: 768-772, 2007.

36. Bofin AM, Ytterhus B and Hagmar BM: TOP2A and HER-2 gene amplification in fine needle aspirates from breast carcinomas. Cytopathology 14: 314-319, 2003.

37. Fasching PA, Weihbrecht S, Haeberle L, Gasparyan A, Villalobos IE, Ma Y, Ekici AB, Wachter DL, Hartmann A, Beckmann MW, et al: HER2 and TOP2A amplification in a hospital-based cohort of breast cancer patients: Associations with patient and tumor characteristics. Breast Cancer Res Treat 145: 193-203, 2014.

38. Martín-Subero JI, Harder L, Gesk S, Schoch R, Novo FJ, Grote W, Calasanz MJ, Schlegelberger B and Siebert R: Amplification of ERBB2, RARA, and TOP2A genes in a myelodysplastic syndrome transforming to acute myeloid leukemia. Cancer Genet Cytogenet 127: 174-176, 2001.

39. Murphy AJ, Hughes CA, Barrett C, Magee H, Loftus B, O'Leary JJ and Sheils O: Low-level TOP2A amplification in prostate cancer is associated with HER2 duplication, androgen resistance, and decreased survival. Cancer Res 67: 2893-2898, 2007.

40. Schaefer-Klein JL, Murphy SJ, Johnson SH, Vasmatzis G and Kovtun IV: Topoisomerase 2 alpha cooperates with androgen receptor to contribute to prostate cancer progression. PLoS One 10: e0142327, 2015

41. Champoux JJ: DNA topoisomerases: Structure, function, and mechanism. Annu Rev Biochem 70: 369-413, 2001.

42. Rhodes DR, Yu J, Shanker K, Deshpande N, Varambally R, Ghosh D, Barrette T, Pandey A and Chinnaiyan AM: Large-scale meta-analysis of cancer microarray data identifies common transcriptional profiles of neoplastic transformation and progression. Proc Natl Acad Sci USA 101: 9309-9314, 2004.

43. Xia L, Wang M, Li H, Tang X, Chen F and Cui J: The effect of aberrant expression and genetic polymorphisms of Rad21 on cervical cancer biology. Cancer Med: May 24, 2018 (Epub ahead of print). doi: 10.1002/cam4.1592.

44. Ahn JH, Kim TJ, Lee JH and Choi JH: Mutant p53 stimulates cell invasion through an interaction with $\operatorname{Rad} 21$ in human ovarian cancer cells. Sci Rep 7: 9076, 2017.

45. Yamamoto G, Irie T, Aida T, Nagoshi Y, Tsuchiya R and Tachikawa T: Correlation of invasion and metastasis of cancer cells, and expression of the RAD21 gene in oral squamous cell carcinoma. Virchows Arch 448: 435-441, 2006.

46. Tessitore A, Cicciarelli G, Del Vecchio F, Gaggiano A, Verzella D, Fischietti M, Vecchiotti D, Capece D, Zazzeroni F and Alesse E: MicroRNAs in the DNA damage/repair network and cancer. Int J Genomics 2014: 820248, 2014.

47. Deb S, Xu H, Tuynman J, George J, Yan Y, Li J, Ward RL, Mortensen N, Hawkins NJ, McKay MJ, et al: RAD21 cohesin overexpression is a prognostic and predictive marker exacerbating poor prognosis in KRAS mutant colorectal carcinomas. Br J Cancer 110: 1606-1613, 2014.

This work is licensed under a Creative Commons Attribution-NonCommercial-NoDerivatives 4.0 International (CC BY-NC-ND 4.0) License. 